\title{
New Upper Bounds for the Heights of Some Light Subgraphs in 1-Planar Graphs with High Minimum Degreefl
}

\author{
Xin Zhang $\quad$ Jian-Liang Wu非 Guizhen Liu|
}

School of Mathematics, Shandong University, Jinan 250100, P. R. China

received $5^{\text {th }}$ May 2010, revised $25^{\text {th }}$ July 2011, accepted $1^{\text {st }}$ September 2011.

A graph is 1-planar if it can be drawn on the plane so that each edge is crossed by at most one other edge. In this paper, it is shown that each 1-planar graph of minimum degree 6 contains a copy of 4-cycle with all vertices of degree at most 19. In addition, we also show that the complete graph $K_{4}$ is light in the family of 1-planar graphs of minimum degree 7, with its height at most 11.

Keywords: 1-planar graphs, light graphs, height.

\section{Introduction}

Throughout this paper, all graphs are finite, simple and undirected. We use $V(G), E(G), \delta(G)$ and $\Delta(G)$ to denote the vertex set, the edge set, the minimum degree and the maximum degree of a graph $G$. Let $e(G)=|E(G)|$ and $v(G)=|V(G)|$. For planar graphs, we use $F(G)$ to denote the face set of $G$ and let $f(G)=|F(G)|$. A vertex (face) of degree $k$ is called a $k$-vertex ( face) and a vertex (face) of degree at least $k$ is called a $k^{+}$-vertex (face). For undefined concepts we refer the reader to [2].

Let $\mathscr{G}$ be a family of graphs and let $H$ be a connected graph such that each member of $\mathscr{G}$ contains a subgraph isomorphic to $H$. Denote $h(H, \mathscr{G})$ and $w(H, \mathscr{G})$ respectively to be the smallest integers with the property that each graph $G \in \mathscr{G}$ contains a subgraph $K \simeq H$ such that $\max _{x \in V(K)}\left\{d_{G}(x)\right\} \leq h(H, \mathscr{G})$ and $\sum_{x \in V(K)}\left\{d_{G}(x)\right\} \leq w(H, \mathscr{G})$. These two parameters $h(H, \mathscr{G})$ and $w(H, \mathscr{G})$ are called the height and the weight of $H$ in the family $\mathscr{G}$. If they are finite, then we say $H$ is light in $\mathscr{G}$, otherwise we say $H$ is heavy in $\mathscr{G}$.

A graph is 1-planar if it can be drawn on the plane so that each edge is crossed by at most one other edge. The notion of 1-planar was introduced by Ringel [12] while studying the simultaneous vertex-face coloring of planar graphs; in the mentioned paper, he proved that each 1-planar graph is 7-colorable. Now this bound was improved to 6 (being sharp) by Borodin [3, 4]. Borodin et al. [5] also proved that each 1-planar graph is acyclically 20-colorable. In addition, the list analogue of vertex coloring of 1-planar

\footnotetext{
†Supported by NSFC grants 10971121, 11101243, 10871119, RFDP grant 20100131120017 and GIIFSDU grant yzc10040.

‡E-mail: sdu.zhang@yahoo.com.cn, xinzhang@mail.sdu.edu.cn

§E-mail: jlwulasdu. edu. cn

TE-mail: gzliu@sdu.edu.cn
}

1365-8050 (C) 2011 Discrete Mathematics and Theoretical Computer Science (DMTCS), Nancy, France 
graphs was investigated in [1] by Albertson and Mohar. In [14], Wang and Lih proved that each 1-planar graph is list 7-colorable. Recently, Zhang et al. showed that each 1-planar graph $G$ with maximum degree $\Delta$ is $\Delta$-edge colorable if $\Delta \geq 10$ [17], or $\Delta \geq 9$ and $G$ contains no chordal 5-cycles [16], or $\Delta \geq 8$ and $G$ contains no chordal 4-cycles [15], or $\Delta \geq 7$ and $G$ contains no 3-cycles [19]; it is $\Delta$-edge choosable and $(\Delta+1)$-total choosable if $\Delta \geq 21$ [20]; it is $(\Delta+1)$-edge choosable and $(\Delta+2)$-total choosable if $\Delta \geq 16$ [20]. The generalization of the total coloring of 1-planar graphs, the so called $(p, 1)$-total labelling problem, is studied in [22].

Although 1-planar graphs is an interesting family, it is still little explored comparing to the family of planar graphs. In general, the family of 1-planar graphs has many fundamental aspects which are different from the planar ones. It is well-known that the planarity test for a graph is polynomial. But for 1-planarity, it is extremely unlucky that its recognizing is NP-complete [10]. This hardness may come from the fact that the set of 1-planar graphs is not closed under taking minors and thus the 1-planarity cannot be characterized by forbidding some minors.

On the other hand, 1-planar graphs also have some similar local structures as planar graphs. For example, the maximum possible number of edges in a 1-planar graph $G$ is $4 v(G)-8$ and there are many 1-planar graphs that attain this bound [7]. This implies that the maximum possible minimum degree of a 1-planar graph is 7. Note that every planar graph is 5-degenerate. So each 1-planar graph with minimum degree 6 or 7 cannot be planar. However, such 1-planar graphs may have many nice and similar properties as planar graphs. In particular, we wonder whether a 1-planar graph with minimum degree 6 or 7 has a similar behavior as a planar graph with high minimum degree concerning the local structures.

In the Fifth Workshop of "Graph Embeddings and Maps on Surfaces" held at Tále in the Summer of 2009, Madaras [11] reported some new results on the light subgraphs in 1-planar graphs with minimum degree 6 and 7, as well as in 1-planar graphs with minimum degree 5 and girth 4. They (Hudák and Madaras) proved that each 1-planar graph with minimum degree 6 contains a 4-cycle with all vertices of degree at most 71, and this upper bound was later decreased to 47 [9]. They also proved the existence of a complete graph $K_{4}$ with all vertices of degree at most 13 in 1-planar graphs with minimum degree 7 [9, [1]. Some other local structures were extensively studied by many authors including [6],8, 10, 13, 18, 21].

In this paper, we improve Hudák and Madaras's upper bounds for the heights of $C_{4}$ and $K_{4}$ in the family of 1-planar graphs with minimum degree 6 and 7, respectively. We prove

Theorem 1 Each 1-planar graph with minimum degree 6 contains a copy of $C_{4}$ with all vertices of degree at most 19.

Theorem 2 Each 1-planar graph with minimum degree 7 contains a copy of $K_{4}$ with all vertices of degree at most 11 .

However, we still do not know whether or not these bounds in Theorems 1 and 2 are best possible. We leave this as an open problem for further research.

\section{Preliminaries}

In the rest of this paper, we always assume that $G$ is a 1-planar graph with minimum degree 6 or 7, and it is drawn on a plane so that (1) every edge is crossed by at most one another edge and (2) the number of crossings is as small as possible. The associated plane graph $G^{\times}$of $G$ is the plane graph that is obtained from $G$ by turning all crossings of $G$ into new 4 -valent vertices. By (1), no two 4-vertices are 
adjacent in $G^{\times}$. A 3-face in $G^{\times}$is called true if it is not incident with 4-vertices and false otherwise. A big face denotes a face of degree at least 4.

For convenience, we use some specialized notations during the proofs in the next section. Let $v$ be a 4-vertex in $G^{\times}$and let $v_{1}, v_{2}, v_{3}, v_{4}$ be its neighbors in clockwise order. By $f_{i}$ denote the face incident with $v v_{i}$ and $v v_{i+1}$ in $G^{\times}$, where the subtraction and addition on subscripts are taken modulo 4 . We call a 4-vertex $v$ closed if $d\left(f_{1}\right)=d\left(f_{2}\right)=d\left(f_{3}\right)=d\left(f_{4}\right)=3$, and open otherwise. It is easy to see that if $d\left(f_{i}\right)=3$, then $v_{i} v_{i+1} \in E\left(G^{\times}\right)$. In this case, let $f_{i}^{\prime}$ be the other face incident with the edge $v_{i} v_{i+1}$. If $d\left(f_{i}^{\prime}\right)=3$, then the third vertex on the boundary of $f_{i}^{\prime}$, which is different from $v_{i}$ and $v_{i+1}$, will be denoted by $v_{i}^{\prime}$. So $v_{i}^{\prime}$ is a 4-vertex if and only if $f_{i}^{\prime}$ is false, in which case we denote the neighbor of $v_{i}$ (or $v_{i+1}$ ) in $G$, such that the edge connecting them in $G$ contains the crossing point $v_{i}^{\prime}$, to be $v_{i}^{\prime \prime}$ (or $v_{i+1}^{\prime \prime}$, respectively). Namely, $v_{i} v_{i}^{\prime \prime}$ and $v_{i+1} v_{i+1}^{\prime \prime}$ are two edges in $G$ that cross each other at the point $v_{i}^{\prime}$. Denote the face incident with the path $x_{i} x_{i}^{\prime} x_{i+1}^{\prime \prime}$ (or $x_{i+1} x_{i}^{\prime} x_{i}^{\prime \prime}$ ) in $G^{\times}$by $f_{i}^{L}$ (or $f_{i}^{R}$, respectively).

The proofs of the two theorems are carried out by contradiction and their beginnings are just the same. Suppose that $G$ is a counterexample to the theorem. Consider the associated plane graph $G^{\times}$of $G$. Using the well-known Euler formula $v\left(G^{\times}\right)-e\left(G^{\times}\right)+f\left(G^{\times}\right)=2$ and the relation $\sum_{v \in V\left(G^{\times}\right)} d(v)=$ $\sum_{f \in F\left(G^{\times}\right)} d(v)=2 e\left(G^{\times}\right)$, we have

$$
\sum_{v \in V\left(G^{\times}\right)}(2 d(v)-10)+\sum_{f \in F\left(G^{\times}\right)}(3 d(f)-10)=-20 .
$$

Define an initial charge $w$ on $V\left(G^{\times}\right) \bigcup F\left(G^{\times}\right)$by $w(v)=2 d(v)-10$ if $v \in V\left(G^{\times}\right)$and $w(f)=$ $3 d(f)-10$ if $f \in F\left(G^{\times}\right)$. Thus we have $\sum_{x \in V\left(G^{\times}\right) \cup F\left(G^{\times}\right)} w(x)=-20$. In order to prove Theorem 1 and Theorem 2, we shall design some discharging rules so that after discharging the new charge $w^{\prime}(x)$ of every element $x \in V\left(G^{\times}\right) \cup F\left(G^{\times}\right)$is nonnegative. Since our rules only move charge around and do not affect the total charges, this leads to a contradiction at the end and completes the proofs.

\section{Proofs}

\subsection{Proof of Theorem 1}

The proof follows the strategy described in Section 2. Suppose that $G$ is a counterexample to Theorem 1 . Then each copy of $C_{4}$ contained in $G$ has a vertex of degree at least 20; we call such a vertex big. The vertices of degree from 6 to 19 are called intermediate vertices. In the following. we proceed with the discharging method and the initial charges are redistributed according to the following rules. Note that all the faces as mentioned in the rules are faces in $G^{\times}$.

R1. Each $6^{+}$-vertex sends $\frac{1}{3}$ to each of its incident faces.

R2. Each 4 -vertex sends $\frac{1}{3}$ to each of its incident false 3 -faces.

R3. Each big face sends 1 to each of its incident 4 -vertices.

R4. Let $\alpha=[x y z]$ be a true 3-face having a common edge $y z$ with a false 3-face $\beta=[u y z]$. If $x$ is a big vertex, then $x$ sends $\frac{2}{3}$ to $u$ through $y z$.

R5. Let $\alpha=[x y z]$ and $\beta=[u y z]$ be two adjacent false 3 -faces and $z$ be a 4-vertex. If $y$ is a big vertex, then $y$ sends 2 to $z$.

R6. Let $\alpha=[x y z]$ be a false 3 -face having a common edge $y z$ with a big face $\beta$. If $y$ is a big vertex and $z$ is a 4 -vertex, then $y$ sends 1 to $z$. 
R7. Let $\alpha=[x y z]$ and $\beta=[u y z]$ be two adjacent false 3-faces, $x$ be a big vertex, $z$ be a 4-vertex, and $y, u$ be two intermediate vertices. Suppose that $y u$ is incident with another false 3-face $\gamma=[y u w]$ and $y y^{\prime}$ crosses $u u^{\prime}$ in $G$ at $w$. If $y u^{\prime} \in E(G)$ and $u^{\prime}$ is an intermediate vertex, or $y^{\prime} u \in E(G)$ and $y^{\prime}$ is an intermediate vertex, then $x$ sends $\frac{1}{3}$ to $w$ through $y z$ and $y u$.

R8. Let $\alpha$ be a big face having a common edge $x y$ with a false 3 -face $\beta=[x y z]$. If $z$ is a 4-vertex, then $\alpha$ sends $\frac{2}{3}$ to $z$ through $x y$.

R9. Let $\alpha$ be a big face having a common edge $x y$ with a false 3 -face $\beta=[x y z]$. If $x$ is a 4-vertex, $y, z$ are both intermediate vertices, and $y z$ is incident with another false 3-face $\gamma=[y z u]$, then $\alpha$ sends $\frac{1}{3}$ to $u$ through $x y$ and $y z$.

By the above rules, one can prove the following two claims that are quite useful.

Claim 1. For a big face $f$, if $R 3$ is applied to $f$ once, then $R 9$ can be executed at most once.

Proof. Suppose that $f$ is a big face with three incident vertices $x, y, z$ such that $x y, y z \in E\left(G^{\times}\right)$and $y$ is a 4-vertex. Then $x z \notin E(G)$ and we can suppose that $x x^{\prime}$ crosses $z z^{\prime}$ in $G$ at $y$. By R3, there must be a transfer from $f$ to $y$ now. If R3 is applied to $f$ once and R9 is executed at least twice, then $x z^{\prime}, x^{\prime} z \in E(G)$ and all of $x, x^{\prime}, z, z^{\prime}$ are intermediate vertices. This implies the existence of a 4-cycle $x x^{\prime} z z^{\prime}$ in $G$ with all its vertices intermediate, a contradiction. Hence R9 can be executed at most once.

Claim 2. For a big vertex $x$, if $R 5$ is applied to $x$ once, then $R 7$ can be executed at most once.

Proof. Let $x y z$ be a false 3-face with a big vertex $x$ and a 4-vertex $z$. Suppose that $x x^{\prime}$ crosses $y y^{\prime}$ in $G$ at $z$. If R5 is applied to $x$ once, then $x y^{\prime} \in E(G)$. Now we prove that R7 cannot be executed twice. For otherwise, $x^{\prime} y z, x^{\prime} y^{\prime} z$ are both false 3 -faces and $y, y^{\prime}, x^{\prime}$ are all intermediate vertices. Furthermore, $x^{\prime} y$ is incident with another 3-face $x^{\prime} y u$ such that $u \neq y^{\prime}$ and $u$ is an intermediate vertex by R7. Consequently, the four intermediate vertices $x^{\prime}, y^{\prime}, y, u$ form a 4-cycle in $G$, a contradiction.

We now check that the final charge of every vertex and face in $G^{\times}$is nonnegative.

Case 1. Suppose that $f$ is a 3 -face. Then by $\mathrm{R} 1$ and $\mathrm{R} 2$, each vertex incident with $f$ sends $\frac{1}{3}$ to $f$. This implies that $w^{\prime}(f)=w(f)+3 \times \frac{1}{3}=-1+1=0$.

Case 2. Suppose that $f$ is a big face. Let $a$ be the number of transfers from $f$ to its incident 4-vertices by R3 and let $b$ be the number of transfers from $f$ to its non-incident 4-vertices by R8. Since no two 4-vertices are adjacent in $G^{\times}, a \leq\left\lfloor\frac{d(f)-b}{2}\right\rfloor$ and $f$ is incident with at least $\left\lceil\frac{d(f)}{2}\right\rceil 6^{+}$-vertices. By Claim 1 , if there is a transfer of 1 from $f$ by R 3 , then there is a total transfer at most $\frac{1}{3}$ from $f$ by $R 9$. Therefore, $w^{\prime}(f) \geq w(f)+\frac{1}{3}\left\lceil\frac{d(f)}{2}\right\rceil-\left(1+\frac{1}{3}\right) a-\frac{2}{3} b \geq \frac{19}{6} d(f)-10-\frac{2}{3}(2 a+b) \geq \frac{19}{6} d(f)-10-\frac{2}{3} d(f)=$ $\frac{5 d(f)-20}{2} \geq 0$ by R1 for $d(f) \geq 4$.

Case 3. Suppose that $v$ is a 4-vertex. Then $w(v)=2 d(v)-10=-2$.

Subcase 3.1. Suppose that $v$ is incident with at least three big faces. Then by R2 and R3, $w^{\prime}(v) \geq$ $w(v)-\frac{1}{3}+3 \times 1=\frac{2}{3}>0$.

Subcase 3.2. Suppose that $v$ is incident with two big faces and two false 3-faces. We follow the definitions in Section 2 and split our proofs into two subcases.

Subcase 3.2.1. Without loss of generality, suppose that $f_{1}, f_{3}$ are big faces and $f_{2}, f_{4}$ are false 3 -faces. Then $\alpha=\left[v_{1} v_{3} v_{2} v_{4}\right]$ is a 4-cycle and thus at least one of the vertices on the boundary of $\alpha$, say $v_{1}$, is a big vertex. This implies that $v$ will receive 1 from $v_{1}$ by R6. Finally, by R2 and R3, we also have $w^{\prime}(v) \geq w(v)+1+2 \times 1-2 \times \frac{1}{3}=\frac{1}{3}>0$.

Subcase 3.2.2. In this case, we suppose that $f_{1}, f_{2}$ are big faces and $f_{3}, f_{4}$ are false 3 -faces. If $v$ is adjacent to at least two big vertices, then at least one of them will send charge to $v$ by R6. Thus we still have $w^{\prime}(v) \geq w(v)+1+2 \times 1-2 \times \frac{1}{3}=\frac{1}{3}>0$ by $\mathbf{R} 2, \mathbf{R} 3$ and R6. So we suppose that $v$ is adjacent to 
at most one big vertex. If one of $v_{1}, v_{3}$ and $v_{4}$ is big, then the redistributed charge of $v$ can be calculated as before, hence, it is nonnegative. If none of $v_{1}, v_{3}$ and $v_{4}$ is big (namely, all of them are intermediate), then $v_{2}$ will not send charge to $v$ by any of the rules no matter $v_{2}$ is big or intermediate. Now, consider the face $f_{3}^{\prime}$. If it is a big face, then it will send $\frac{2}{3}$ to $v$ through $v_{3} v_{4}$ by R8. If $f_{3}^{\prime}$ is 3 -face, denoted by $v_{3} v_{4} v_{3}^{\prime}$ (note that $v_{3}^{\prime} \neq v_{1}, v_{2}$ ), then $v_{3}^{\prime}$ must be a big vertex while $f_{3}^{\prime}$ is true, that is because $v_{1} v_{3} v_{3}^{\prime} v_{4}$ forms a 4-cycle in $G$ with $v_{1}, v_{3}, v_{4}$ being intermediate. In such a case, $v_{3}^{\prime}$ will send $\frac{2}{3}$ to $v$ through $v_{3} v_{4}$ by R4. If $f_{3}^{\prime}$ is false, then $v_{3}^{\prime}$ is a 4-vertex. Now consider the faces $f_{3}^{L}$ and $f_{3}^{R}$. If $f_{3}^{L}$ is big face, then it will send $\frac{1}{3}$ to $v$ through $v_{3} v_{4}$ by R9. Otherwise $f_{3}^{L}$ must be a false 3 -face, denoted by $v_{3} v_{3}^{\prime} v_{4}^{\prime \prime}$ (note that $v_{4}^{\prime \prime} \neq v_{1}, v_{2}$ ). In this case $v_{1} v_{3} v_{4}^{\prime \prime} v_{4}$ is a 4-cycle in $G$ with $v_{1}, v_{3}, v_{4}$ being intermediate, which implies that $v_{4}^{\prime \prime}$ must be a big vertex. Then by R7, $v_{4}^{\prime \prime}$ will send $\frac{1}{3}$ to $v$ through $v_{3} v_{4}$. By a similar discussion on $f_{3}^{R}, v$ will receive another $\frac{1}{3}$ from $f_{3}^{R}$ or $v_{3}^{\prime \prime}$ through $v_{3} v_{4}$ (again, note that $v_{3}^{\prime \prime} \neq v_{1}$, and if $v_{3}^{\prime \prime}=v_{2}$, then $f_{3}^{R}$ must be a big face). Consequently, using the transfers through $v_{3} v_{4}, v$ receives totally $2 \times \frac{1}{3}=\frac{2}{3}$. By symmetry, the same discussion can be applied to the calculation of the transfers through $v_{1} v_{4}$. Thus, by R2 and R3, we still have $w^{\prime}(v) \geq w(v)+2 \times 1-2 \times \frac{1}{3}+2 \times \frac{2}{3}=\frac{2}{3}>0$.

Subcase 3.3. Suppose that $v$ is incident with one big faces and three false 3 -faces. Without loss of generality, we assume that $f_{1}$ is big and the other three faces are false. Since $v_{1} v_{3} v_{4} v_{2}$ forms a 4-cycle in $G, v$ must be adjacent to at least one big vertex. If $v$ is adjacent to at least two big vertices, then by $\mathrm{R} 5$ and R6, $v$ receives at least $2 \times 1=2$ from its big neighbors. Together with R2 and R3, we have $w^{\prime}(v) \geq w(v)+1-3 \times \frac{1}{3}+2=0$. Now we consider the case when $v$ is adjacent to exactly one big vertex. If $v_{3}$ or $v_{4}$ is big, then by R5, a transfer of 2 from $v_{3}$ or $v_{4}$ to $v$ will be put into practice. This implies that $w^{\prime}(v) \geq w(v)+1-3 \times \frac{1}{3}+2=0$. If $v_{1}$ or $v_{2}$ is big, say $v_{2}$, then $v_{2}$ will send 1 to $v$ by R6. By the same argument as in Subcase 3.2.2, $v$ will also receive $\frac{2}{3}$ through the edge $v_{3} v_{4}$ and another $\frac{2}{3}$ through the edge $v_{1} v_{4}$. In total, by R2 and R3, we shall also have $w^{\prime}(v) \geq w(v)+1-3 \times \frac{1}{3}+1+2 \times \frac{2}{3}=\frac{1}{3}>0$.

Subcase 3.4. Suppose that $v$ is incident with four false 3 -faces. Since $v_{1} v_{2} v_{3} v_{4}$ is a 4-cycle in $G, v$ must be adjacent to at least one big vertex. If $v$ is adjacent to at least two big vertices, then by R2 and R5, we have $w^{\prime}(v) \geq w(v)-4 \times \frac{1}{3}+2 \times 2=\frac{2}{3}>0$. If $v$ is adjacent to exactly one big vertex, say $v_{2}$, then $v_{2}$ will send 2 to $v$ by R5. Again, by the same argument as in Subcase 3.2.2, $v$ will totally receive $2 \times \frac{2}{3}=\frac{4}{3}$ through the edges $v_{1} v_{4}$ and $v_{3} v_{4}$. This implies that $w^{\prime}(v) \geq w(v)-4 \times \frac{1}{3}+2+\frac{4}{3}=0$ by R2 and R3.

Case 4. Suppose that $v$ is an intermediate vertex. Then $v$ sends out charges only by R1, which implies that $w^{\prime}(v) \geq w(v)-\frac{1}{3} d(v)=2 d(v)-10-\frac{1}{3} d(v)=\frac{5 d(v)-30}{3} \geq 0$ for $d(v) \geq 6$.

Case 5. Suppose that $v$ is a big vertex. Let $F$ denote the subgraph induced by the faces which are incident with $v$. Then $F$ can be decomposed into many parts, each of which is one of the following five clusters in Figure 1. and any two parts of which are adjacent only if they have a common edge $v w$ such that $w$ is a $6^{+}$-vertex. The hollow vertices in Figure 1 are 4-vertices and the solid ones are $6^{+}$-vertices; all the faces marked by $4^{+}$are big faces and there is at least one big face contained in the clusters of type 2, 4 and 5 .

Let $n_{i}$ be the number of clusters of type $i$ contained in $F$. By their definitions, one can easily observe that $2 n_{1}+2 n_{2}+n_{3}+3 n_{4}+n_{5} \leq d(v)$. If there is a cluster of type 1 in $F$, then $v$ will send 2 to $y$ by R5. Meanwhile, $v$ will also send out at most $\frac{1}{3}$ through either $x y$ or $y z$ by $\mathrm{R} 7$ and Claim 2. If there is a cluster of type 2 in $F$, then $v$ will send 1 to $y$ by R6 and send out at most $\frac{1}{3}$ through $x y$ by R7. If there is a cluster of type 3 in $F$, then $v$ will send out at most $\frac{2}{3}$ though $x y$ by R4. If there is a cluster of type 4 in $F$, then $v$ will send 1 to each of $y$ and $u$ by R6 and totally send out at most $2 \times \frac{1}{3}=\frac{2}{3}$ through $x y$ and $u w$ by R7. If there is a cluster of type 5 in $F$, then $v$ will not send out charges via this cluster by any of the rules. Therefore, together with R1, we have $w^{\prime}(v) \geq w(v)-\frac{1}{3} d(v)-\left(2+\frac{1}{3}\right) n_{1}-\left(1+\frac{1}{3}\right) n_{2}-\frac{2}{3} n_{3}-\left(2 \times 1+\frac{2}{3}\right) n_{4}=$ 

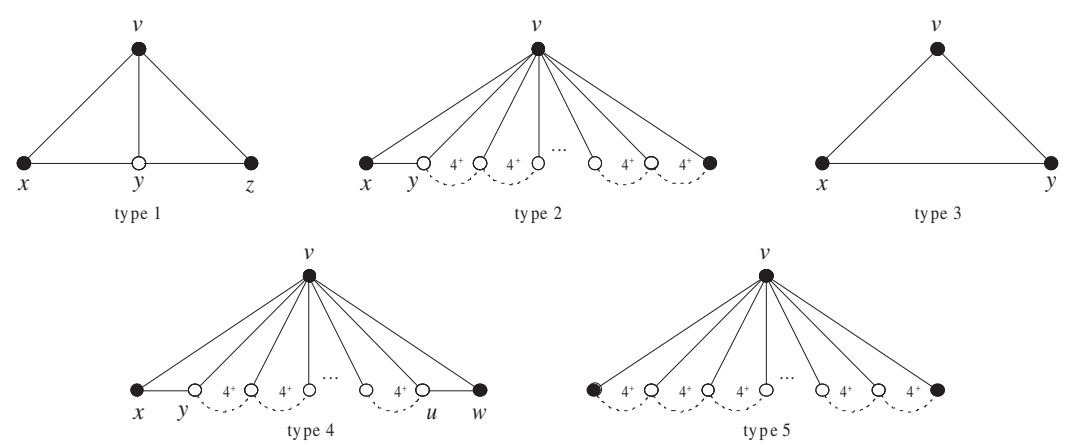

Fig. 1: Five types of cluster

$\frac{5}{3} d(v)-10-\frac{7}{6}\left(2 n_{1}+2 n_{2}+n_{3}+3 n_{4}+n_{5}\right)+\left(n_{2}+\frac{1}{2} n_{3}+\frac{5}{6} n_{4}+\frac{7}{6} n_{5}\right) \geq \frac{5}{3} d(v)-10-\frac{7}{6} d(v)=\frac{d(v)-20}{2} \geq 0$ for $d(v) \geq 20$.

\subsection{Proof of Theorem 2}

The proof also follows the strategy described in Section 2. Let $G$ be a counterexample to Theorem 2 So each copy of $K_{4}$ in $G$ contains a $12^{+}$-vertex, which is called big vertex. The vertices of degree from 7 to 11 are called intermediate vertices. Let $t(v)$ be the number of 3-faces that are incident with $v$. Then $v$ is closed if and only if $t(v)=4$ (recall the definition of closed vertices in Section 2). Let $c(v)$ (or $c(f)$ ) be the number of 4-vertices that are adjacent to (or incident with) $v$ (or $f$ ). By $c_{1}(v)$ and $c_{2}(v)$, respectively, we denote the number of closed 4-vertices and open 4-vertices adjacent to $v$. Obviously we have $c_{1}(v)+c_{2}(v)=c(v)$.

Claim 1. If $d(v)=7$, then $t(v) \leq 6$ if $c(v)=4, t(v) \leq 4$ if $c(v)=5, t(v) \leq 2$ if $c(v) \geq 6$.

Proof. This follows directly from the fact that no two 4-vertices are adjacent in $G^{\times}$.

Claim 2. If $d(v) \geq 8$, then $t(v)+c(v) \leq \frac{3}{2} d(v)$ and $t(v)+2 c(v) \leq 2 d(v)$.

Proof. If $c(v) \leq\left\lfloor\frac{d(v)}{2}\right\rfloor$, then this holds trivially. So we assume that $c(v) \geq\left\lceil\frac{d(v)}{2}\right\rceil$. Beginning with $\left\lceil\frac{d(v)}{2}\right\rceil$, each unit of increase on $c(v)$ will imply two units of decrease on the largest possible number of the 3-faces that are incident with $v$, that is because no two 4-vertices are adjacent in $G^{\times}$. Therefore, $t(v) \leq d(v)-2\left(c(v)-\left\lceil\frac{d(v)}{2}\right\rceil\right)$ if $d(v)$ is even, and $t(v) \leq d(v)-1-2\left(c(v)-\left\lceil\frac{d(v)}{2}\right\rceil\right)$ if $d(v)$ is odd. In either case, $t(v)+2 c(v) \leq 2 d(v)$. Since $c(v) \geq \frac{d(v)}{2}$, we have $t(v)+c(v) \leq 2 d(v)-c(v) \leq \frac{3}{2} d(v)$.

Claim 3. If $d(v) \geq 12$, then $2 c_{1}(v)+c_{2}(v) \leq d(v)$.

Proof. Since no two 4-vertices are adjacent in $G^{\times}$, we have $c_{1}(v) \leq\left\lfloor\frac{d(v)}{2}\right\rfloor$. If $c(v) \leq\left\lfloor\frac{d(v)}{2}\right\rfloor$, then $2 c_{1}(v)+c_{2}(v)=c_{1}(v)+c(v) \leq d(v)$. If $c(v)>\left\lfloor\frac{d(v)}{2}\right\rfloor$, then each unit of increase on $c(v)$ from $\left\lfloor\frac{d(v)}{2}\right\rfloor$ implies the existence of at least two open 4-vertices that are adjacent to $v$. This implies that $c_{2}(v) \geq 2\left(c(v)-\left\lfloor\frac{d(v)}{2}\right\rfloor\right) \geq 2\left(c_{1}(v)+c_{2}(v)\right)-d(v)$. Therefore, we have $2 c_{1}(v)+c_{2}(v) \leq d(v)$.

We complete the proof of Theorem 2 by applying the following rules of discharging.

R1. Each big face sends 1 to each of its incident 4 -vertices.

R2. Each intermediate vertex sends $\frac{1}{3}$ to each of its adjacent 4 -vertices. 
R3. Each big vertex sends $\frac{1}{3}$ to each of its adjacent open 4 -vertices, and $\frac{13}{9}$ to each of its adjacent closed 4-vertices.

R4. Let $\alpha=[x y z]$ be a true 3 -face. Then each of $x, y, z$ sends $\frac{1}{3}$ to $\alpha$.

R5. Let $\alpha=[x y z]$ be a false 3-face. If $x$ is a 4-vertex, then $x$ sends $\frac{1}{9}$ while both $y$ and $z$ sends $\frac{4}{9}$ to $\alpha$.

We now check that the final charge of every vertex and face in $G^{\times}$is nonnegative.

Case 1. Suppose that $f$ is a 3-face. By R4 and R5, one can observe that $w^{\prime}(f)=w(f)+1=-1+1=0$.

Case 2. Suppose that $f$ is a big face. Since no two 4-vertices are adjacent in $G^{\times}, c(f) \leq\left\lfloor\frac{d(f)}{2}\right\rfloor$. Therefore, by R1, we have $w^{\prime}(f) \geq w(f)-\left\lfloor\frac{d(f)}{2}\right\rfloor \geq \frac{5 d(f)-20}{2} \geq 0$ for $d(f) \geq 4$.

Case 3. Suppose that $v$ is a 4-vertex. If $v$ is incident with at least two big faces, then by R1, R2, R3 and R5, $w^{\prime}(v) \geq w(v)+1 \times 2+4 \times \frac{1}{3}-2 \times \frac{1}{9}=\frac{10}{9}>0$. If $v$ is incident with exactly one big face, then we still have $w^{\prime}(v) \geq w(v)+1+4 \times \frac{1}{3}-3 \times \frac{1}{9}=0$. If $v$ is incident with no big faces, then $v$ is closed. Meanwhile, $v_{1}, v_{2}, v_{3}$ and $v_{4}$ forms a $K_{4}$ in $G$, which implies that at least one of them is a big vertex, say $v_{1}$. Then $v_{1}$ will send $\frac{13}{9}$ to $v$ by R3. Since $v_{2}, v_{3}$ and $v_{4}$ are either intermediate or big, each of them shall send at least $\frac{1}{3}$ to $v$ by R2 and R3. Together with R5, we also have $w^{\prime}(v) \geq w(v)+\frac{13}{9}+3 \times \frac{1}{3}-4 \times \frac{1}{9}=0$. Case 4. Suppose that $v$ is an intermediate vertex.

Subcase 4.1. Suppose that $d(v)=7$. If $c(v) \leq 3$, then $v$ can be incident with at most six false 3 -faces. So by R2, R4 and R5, we have $w^{\prime}(v) \geq w(v)-3 \times \frac{1}{3}-\frac{1}{3}-6 \times \frac{4}{9}=0$. If $c(v)=4$, then by Claim 1 , $t(v) \leq 6$. It follows that $w^{\prime}(v) \geq w(v)-4 \times \frac{1}{3}-6 \times \frac{4}{9}=0$. If $c(v)=5$, then $t(v) \leq 4$ by Claim 1 , which implies that $w^{\prime}(v) \geq w(v)-5 \times \frac{1}{3}-4 \times \frac{4}{9}=\frac{5}{9}>0$. If $c(v) \geq 6$, then $t(v) \leq 2$ by Claim 1 . Consequently, we still have $w^{\prime}(v) \geq w(v)-\frac{1}{3} c(v)-\frac{4}{9} t(v) \geq 4-\frac{7}{3}-\frac{8}{9}=\frac{7}{9}>0$.

Subcase 4.2. Suppose that $8 \leq d(v) \leq 11$. By R2, R4, R5 and Claim 2 , we have $w^{\prime}(v) \geq w(v)-\frac{1}{3} c(v)-$ $\frac{4}{9} t(v) \geq 2 d(v)-10-\frac{4}{9}(c(v)+t(v)) \geq 2 d(v)-10-\frac{4}{9} \times \frac{3}{2} d(v)=\frac{4 d(v)-30}{3}>0$ for $d(v) \geq 8$.

Case 5. Suppose that $v$ is a big vertex. If $c(v) \leq\left\lfloor\frac{d(v)}{2}\right\rfloor$, then by R3, R4 and R5, $w^{\prime}(v) \geq w(v)-\frac{13}{9} c(v)-$ $\frac{4}{9} d(v) \geq 2 d(v)-10-\frac{13}{9} \times \frac{d(v)}{2}-\frac{4}{9} d(v)=\frac{5 d(v)-60}{6} \geq 0$ for $d(v) \geq 12$. On the other hand, if $c(v) \geq$ $\left\lceil\frac{d(v)}{2}\right\rceil$, then by R3, R4, R5, Claim 2 and Claim 3, we have $w^{\prime}(v) \geq w(v)-\frac{13}{9} c_{1}(v)-\frac{1}{3} c_{2}(v)-\frac{4}{9} t(v)=$ $2 d(v)-10-\frac{1}{3}\left(2 c_{1}(v)+c_{2}(v)\right)-\frac{4}{9} t(v)-\frac{7}{9} c_{1}(v) \geq 2 d(v)-10-\frac{1}{3} d(v)-\frac{4}{9}\left(2 d(v)-2 c_{1}(v)-2 c_{2}(v)\right)-$ $\frac{7}{9} c_{1}(v)=\frac{7}{9} d(v)-10+\frac{1}{9} c_{1}(v)+\frac{8}{9} c_{2}(v) \geq \frac{7}{9} d(v)-10+\frac{1}{9} c(v) \geq \frac{7}{9} d(v)-10+\frac{1}{18} d(v)=\frac{5 d(v)-60}{6} \geq 0$ for $d(v) \geq 12$.

\section{References}

[1] M. O. Albertson and B. Mohar. Coloring vertices and faces of locally planar graphs. Graphs Combin., 22:289-295, 2006.

[2] J. A. Bondy and U. S. R. Murty. Graph Theory with Applications. American Elsevier Pub. Co., New York, 1976.

[3] O. V. Borodin. Solution of Ringel's problems on the vertex-face coloring of plane graphs and on the coloring of 1-planar graphs. Metody Diskret. Analiz, 41:12-26, 1984. (in Russian).

[4] O. V. Borodin. A new proof of the 6 color theorem. J. Graph Theory, 19(4):507-521, 1995.

[5] O. V. Borodin, A. V. Kostochka, A. Raspaud, and E. Sopena. Acyclic colouring of 1-planar graphs. Discrete Appl. Math., 104:29-41, 2001. 
[6] O. V. Borodin, I. G. Dmitriev, and A. O. Ivanova. The height of a cycle of length 4 in 1-planar graphs with minimum degree 5 without triangles. Diskretn. Anal. Issled. Oper., 15(1):11-16, 2008. (in Russian).

[7] I. Fabrici and T. Madaras. The structure of 1-planar graphs. Discrete Math., 307:854-865, 2007.

[8] D. Hudák and T. Madaras. On local structures of 1-planar graphs of minimum degree 5 and girth 4. Discuss. Math. Graph Theory, 29:385-400, 2009.

[9] D. Hudák and T. Madaras. On local properties of 1-planar graphs with high minimum degree. Ars Math. Contemp., 4(2):245-254, 2011.

[10] V. P. Korzhik and B. Mohar. Minimal obstructions for 1-immersions and hardness of 1-planarrity test. Lecture Notes in Comput. Sci., 5417:302-312, 2009.

[11] T. Madaras. On local properties of 1-planar graphs with specified minimum degree and girth. In The Fifth Workshop, Graph Embeddings and Maps on Surfaces, Tále, Low Tatras, Slovakia, 2009.

[12] G. Ringel. Ein Sechsfarbenproblem auf der Kugel. Abh. Math. Semin. Univ. Hambg., 29:107-117, 1965. (in German).

[13] Y. Suzuki. Optimal 1-planar graphs which triangulate other surfaces. Discrete Math., 310:6-11, 2010.

[14] W. Wang and K.-W. Lih. Coupled choosability of plane graphs. J. Graph Theory, 58:27-44, 2008.

[15] X. Zhang and G. Liu. On edge colorings of 1-planar graphs without adjacent triangles. Submitted, 2011.

[16] X. Zhang and G. Liu. On edge colorings of 1-planar graphs without chordal 5-cycles. Ars Combin., 2011. (accepted for publication).

[17] X. Zhang and J.-L. Wu. On edge colorings of 1-planar graphs. Inform. Process. Lett., 111(3): 124-128, 2011.

[18] X. Zhang, G. Liu, and J.-L. Wu. Structural properties of 1-planar graphs and an application to acyclic edge coloring. Scientia Sinica Mathematica, 40(10):1025-1032, 2010. (in Chinese).

[19] X. Zhang, G. Liu, and J.-L. Wu. Edge coloring of triangle-free 1-planar graphs. J. Shandong Univ. Nat. Sci., 45(6):15-17, 2010. (in Chinese).

[20] X. Zhang, J.-L. Wu, and G. Liu. List edge and list total coloring of 1-planar graphs. Submitted, 2010.

[21] X. Zhang, G. Liu, and J.-L. Wu. Light subgraphs in the family of 1-planar graphs with high minimum degree. Acta Math. Sin. (Engl. Ser.), 2011. (accepted for publication).

[22] X. Zhang, Y. Yu, and G. Liu. On ( $p, 1)$-total labelling of 1-planar graphs. Cent. Eur. J. Math., 2011. doi: 10.2478/s11533-011-0092-1. 